\title{
Minimal Dark Matter bound states at future colliders
}

\author{
Salvatore Bottaro, ${ }^{a, c}$ Alessandro Strumia ${ }^{b}$ and Natascia Vignaroli ${ }^{b, c}$ \\ ${ }^{a}$ Scuola Normale Superiore, \\ P.za dei Cavalieri, 7, 56126 Pisa PI, Italia \\ ${ }^{b}$ Dipartimento di Fisica, Università di Pisa, \\ Largo Bruno Pontecorvo, 3, 56127 Pisa PI, Italia \\ ${ }^{c}$ INFN, Sezione di Pisa, \\ Largo Bruno Pontecorvo, 3, 56127 Pisa PI, Italia \\ E-mail: salvatore.bottaro@sns.it, alessandro.strumia@unipi.it, \\ natascia.vignaroli@df .unipi.it
}

Abstract: The hypothesis that Dark Matter is one electroweak multiplet leads to predictive candidates with multi- $\mathrm{TeV}$ masses that can form electroweak bound states. Bound states with the same quantum numbers as electroweak vectors are found to be especially interesting, as they can be produced resonantly with large cross sections at lepton colliders. Such bound states exist e.g. if DM is an automatically stable fermionic weak 5-plet with mass $M \approx 14 \mathrm{TeV}$ such that the $\mathrm{DM}$ abundance is reproduced thermally. In this model, a muon collider could resolve three such bound states. Production rates are so large that details of DM spectroscopy can be probed with larger statistics: we compute the characteristic pattern of single and multiple $\gamma$ lines.

Keywords: Beyond Standard Model, Dark matter, $e^{+}-e^{-}$Experiments, Particle and resonance production, Spectroscopy

ARXIV EPRINT: 2103.12766 


\section{Contents}

1 Introduction 1

2 Minimal Dark Matter and its bound states 2

3 Bound-state production at colliders 4

3.1 Production of ${ }^{n} s_{3}$ bound states from $\mu^{-} \mu^{+}$collisions 5

3.2 Production of other bound states from $V V$ collisions $\quad 7$

3.3 Decays of bound states and their collider signals 8

4 Conclusions 10

\section{Introduction}

The hypothesis that Dark Matter is the thermal relic of one new multiplet under the Standard Model gauge group provides some predictive allowed candidates [1]. In particular, the cosmological DM abundance is reproduced for $\mathrm{TeV}$-scale values of the DM mass, above the LHC reach. A fermionic 5-plet under $\mathrm{SU}(2)_{L}$ with zero hypercharge is a particularly interesting possibility, being automatically long lived enough to be DM. Its thermal abundance matches the $\mathrm{DM}$ density for a mass $M \approx 14 \mathrm{TeV}$, after taking into account Sommerfeld and bound-state corrections [2]. Its univocal prediction for direct detection can be tested [1,3], but its production at colliders would allow to measure more than one number. However, even a giant $p p$ collider at $100 \mathrm{TeV}$ would have a limited reach, up to about $4 \mathrm{TeV}$ [4] (see also [5-8]).

A future muon collider could be built in the existing $27 \mathrm{~km} \mathrm{LEP} / \mathrm{LHC}$ circular tunnel. In such a case, $\mu^{ \pm}$beams can reach the maximal energy $\sqrt{s}$ allowed by magnetic fields. This was $\sqrt{s} \approx 14 \mathrm{TeV}$ at LHC, but future magnets can realistically increase it up to $\sqrt{s} \sim 30 \mathrm{TeV}$. Concerning the integrated luminosity, a value $\mathcal{L} \sim 90 /$ ab at $\sqrt{s}=30 \mathrm{TeV}$ is considered possible [9], provided that radiological hazards due to muon decays into neutrinos can be limited.

According to [9], a muon collider with this luminosity cannot probe a Minimal DM 5plet with $M \approx 14 \mathrm{TeV}$, unless experiments are able to find in the beam-related background the tracks left by its charged components, short because produced with non-relativistic velocity (see [10] for possible strategies). Otherwise, missing energy signals tagged by an extra muon or gamma have low cross sections and can only probe 5 -plets lighter than about $10 \mathrm{TeV}[9]$.

We show that extra signals arise taking into account that such DM forms weak bound states with binding energy $E_{B} \sim 100 \mathrm{GeV}$. Such bound states annihilate into SM particles 
(including $\mu^{+} \mu^{-}$, for appropriate bound states with the same quantum numbers of electroweak vectors) with a width, $\Gamma_{B} \sim \alpha_{2}^{5} M$, that is small but not much smaller than the expected energy resolution of a muon collider, $\sigma_{E} \sim 10^{-3} E$. Production and annihilation of DM bound states $B$ thereby results into a large cross section among visible SM particles,

$$
\sigma\left(\mu^{+} \mu^{-} \rightarrow B \rightarrow f \bar{f}\right) \sim \sigma_{\text {peak }} \frac{\Gamma_{B}}{\sigma_{E}} \quad \text { where } \quad \sigma_{\text {peak }} \sim \frac{4 \pi}{s}
$$

is the maximal cross section allowed by unitarity.

One needs to run around the peak, and the minimal 5-plet DM model allows to predict the DM mass, $M \approx 14 \mathrm{TeV}$, from the cosmological DM density. More in general (for example a family of three 5-plets allows for smaller $M$ ) one could first discover DM through other signals, measure its mass, and next run on the peak, possibly gradually reducing the beam energy spread $\sigma_{E}$ of the collider to achieve the maximal cross section. The energy resolution of a muon collider can be reduced by at least one order of magnitude, down to $\sigma_{E} \sim 10^{-4} E$, at the price of proportionally reducing its luminosity. We also consider other bound states and $p p$ colliders, obtaining small cross sections as no resonant production is possible. ${ }^{1}$

The paper is structured as follows. In section 2 we summarize the DM model and the properties of DM bound states. In section 3 we discuss bound state production at colliders, focusing in 3.1 on the main signals from states that can be produced resonantly with large cross section, in 3.2 on other bound states, in 3.3 on rarer but very characteristic signals coming from decays among DM bound states, such as $\gamma$ lines. In section 4 we give conclusions, and mention one more (curious but small) signal of Minimal DM.

\section{Minimal Dark Matter and its bound states}

The SM is extended adding a fermionic 5-plet $\mathcal{X}$ under $\mathrm{SU}(2)_{L}$ with zero hypercharge, such that the most general renormalizable Lagrangian is

$$
\mathscr{L}=\mathscr{L}_{\mathrm{SM}}+\frac{1}{2} \overline{\mathcal{X}}(i \not D+M) \mathcal{X}
$$

The 5-plet contains a Majorana neutral component $\mathcal{X}^{0}$, together with Dirac fermions $\mathcal{X}^{ \pm}$ and $\mathcal{X}^{ \pm \pm}$with charge \pm 1 and \pm 2 . Due to its electric field, the components with charge $q$ are heavier than $\mathcal{X}^{0}$ by $q^{2} \Delta M$, where $\Delta M=\alpha_{2} M_{W} \sin ^{2}\left(\theta_{\mathrm{W}} / 2\right) \approx 166 \mathrm{MeV}$. Thereby charged states decay into the lightest DM-candidate $\mathcal{X}^{0}$.

The components of $\mathcal{X}$ can be pair-produced at collider. Pairs of 5-plets can form Coulombian-like electroweak bound states given that $M \gtrsim M_{W, Z} / \alpha_{2}$. Bound states can be computed in components [18], or more simply in $\mathrm{SU}(2)_{L}$-symmetric approximation, setting $M_{W} \approx M_{Z}$ and neglecting $\Delta M[2]$. States of two quintuplets decompose under $\mathrm{SU}(2)_{L}$ as $5 \otimes 5=1_{S} \oplus 3_{A} \oplus 5_{S} \oplus 7_{A} \oplus 9_{S}$. The attractive channels with potential $V=-\alpha_{\text {eff }} e^{-M_{W, Z} r} / r$ have isospin $I=1\left(\alpha_{\text {eff }}=6 \alpha_{2}\right), I=3\left(\alpha_{\text {eff }}=5 \alpha_{2}\right)$, and $I=5\left(\alpha_{\text {eff }}=3 \alpha_{2}\right)$. The SM weak

\footnotetext{
${ }^{1} \mathrm{DM}$ bound states of an electroweak triplet have been discussed in [11] at a $p p$ collider, where no resonant production is possible. We here include important non-abelian Coulomb-like potentials. See also [12-17].
} 


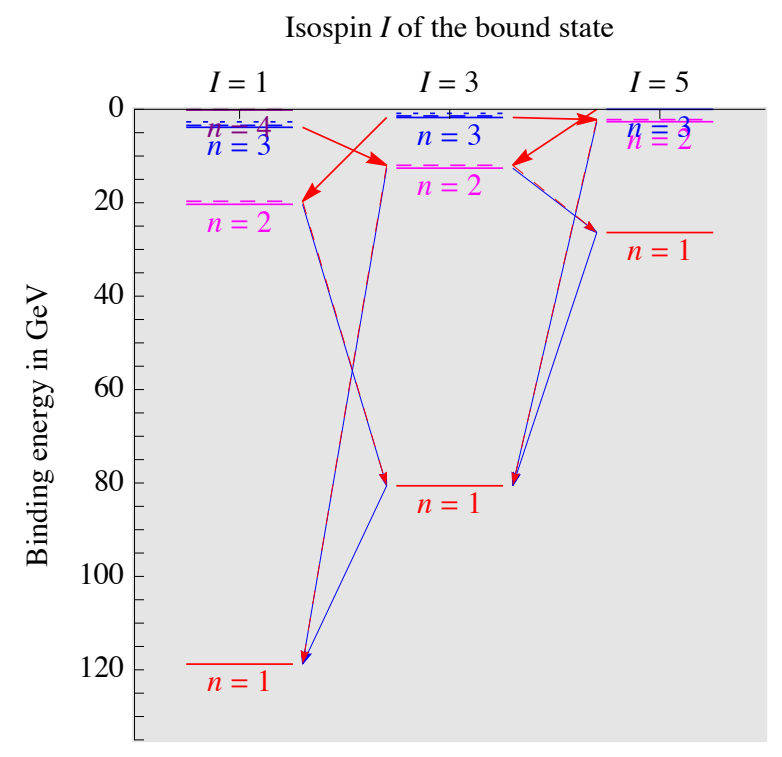

Figure 1. Energy levels of bound states of two Minimal Dark Matter 5-plets with $M=14 \mathrm{TeV}$. Continuous lines have $\ell=0$, dashed lines have $\ell=1$, dotted lines have $\ell=2$. The blue (red) arrows indicate some main magnetic (electric) decays.

couplings renormalized at TeV energy are $\alpha_{2}=1 / 30.8$ and $\alpha_{\mathrm{em}}=1 / 128.6$. The binding energies of bound states can be approximated as [2]

$$
E_{B} \approx \frac{\alpha_{\mathrm{eff}}^{2} M}{4 n^{2}}\left[1-n^{2} y-0.53 n^{2} y^{2} \ell(\ell+1)\right]^{2} \quad \text { where } \quad y \approx \frac{1.74 M_{W, Z}}{\alpha_{\mathrm{eff}} M}
$$

and $\ell$ is angular momentum. The bound state exists only when the term in the squared parenthesis is positive. Furthermore, only bound states with $(-1)^{\ell+S+\tilde{I}}=1$ have the correct fermionic anti-symmetry, where $I=2 \tilde{I}+1$ is the dimension of the representation. As usual for bound states of two fermions, our states have quantum numbers $C=(-1)^{\ell+S}$ under charge conjugation and $P=(-1)^{\ell+1}$ under parity (which gets broken when chiral gauge interactions of SM fermions play a role).

The resulting 5-plet bound states at constituent mass $M=14 \mathrm{TeV}$ are plotted in figure 1 , and table 1 lists their main properties. ${ }^{2}$ Bound states can decay via annihilation of their constituents with rate $\Gamma_{\mathrm{ann}}$, or into deeper states with rate $\Gamma_{\mathrm{dec}}$. Bound states with $J \equiv \ell \oplus S$ equal to 1 or 5 can annihilate into two $\mathrm{SU}(2)_{L}$ vectors $V V$, while vector bound states with $J=3$ cannot because of the Landau-Yang theorem.

We are especially interested in bound states that annihilate into SM fermions, as they can thereby be directly produced in $\mu^{+} \mu^{-}$collisions. Such states are those with the same quantum numbers as the weak vectors $W_{\mu}^{a}$, so that such bound states mix and inherit couplings to fermions. These special bound states are the ${ }_{1}^{n} s_{3}^{--}$vector triplets with $S=1$, $\ell=0$ (the full notation is explained in the first row of table 1), which decay into SM

\footnotetext{
${ }^{2}$ While we agree with the generic formulæ for the rates in [2], we found a missing order one factor in the application to the 5-plet: the decay rates of the $2 p$ states differ from eq. (91) in [2] because a $\alpha_{2}$ should be $\alpha_{\text {eff }}$. These $2 p$ decay rates negligibly affect the cosmological relic abundance computed in [2].
} 


\begin{tabular}{|c|c|c|c|c|c|c|c|c|c|}
\hline \multirow{2}{*}{$\begin{array}{l}\text { name } \\
{ }_{J}^{n} \ell_{I}^{P C}\end{array}$} & \multicolumn{5}{|c|}{ Quantum numbers } & \multicolumn{2}{|l|}{ Annihilation } & \multicolumn{2}{|l|}{ Decay } \\
\hline & $n$ & $I$ & & $\ell$ & $E_{B}$ & $\Gamma_{\text {ann }}$ & into & $\Gamma_{\mathrm{dec}}$ & into \\
\hline${ }_{1}^{1} s_{1}^{-+}$ & 1 & 1 & 0 & 0 & $118 \mathrm{GeV}$ & $3240 \alpha_{2}^{5} M \approx 1.63 \mathrm{GeV}$ & $V \tilde{V}$ & 0 & - \\
\hline${ }_{1}^{1} s_{3}^{--}$ & 1 & 3 & 1 & 0 & $81 \mathrm{GeV}$ & $15625 \alpha_{2}^{5} M / 48 \approx 0.17 \mathrm{GeV}$ & $f_{L} \bar{f}_{L}+H H^{*}$ & $36 \alpha_{2}^{6} \alpha_{\mathrm{em}} M \approx 4.6 \mathrm{keV}$ & ${ }^{1} s_{1} \gamma$ \\
\hline${ }_{1}^{1} s_{5}^{-+}$ & 1 & 5 & 0 & 0 & $26 \mathrm{GeV}$ & $567 \alpha_{2}^{5} M / 4 \approx 0.07 \mathrm{GeV}$ & $V \tilde{V}$ & $295 \alpha_{2}^{6} \alpha_{\mathrm{em}} M \approx 38 \mathrm{keV}$ & ${ }^{1} s_{3} \gamma$ \\
\hline${ }_{1}^{2} s_{1}^{-+}$ & 2 & 1 & & 0 & $20.3 \mathrm{GeV}$ & $405 \alpha_{2}^{5} M \approx 0.2 \mathrm{GeV}$ & $V \tilde{V}$ & $13 \alpha_{2}^{6} \alpha_{\mathrm{em}} M \approx 1.7 \mathrm{keV}$ & ${ }^{1} s_{3} \gamma$ \\
\hline${ }_{1}^{2} s_{3}^{--}$ & 2 & 3 & 1 & 0 & $13 \mathrm{GeV}$ & $15625 \alpha_{2}^{5} M / 384 \approx 21 \mathrm{MeV}$ & $f_{L} \bar{f}_{L}+H H^{*}$ & $\left(6.9 \alpha_{2}+0.3 \alpha_{\mathrm{em}}\right) \alpha_{2}^{6} M \approx 3.7 \mathrm{keV}$ & ${ }^{1} s_{1+5} V$ \\
\hline${ }_{1}^{2} s_{5}^{-+}$ & 2 & 5 & 0 & 0 & $2.6 \mathrm{GeV}$ & $567 \alpha_{2}^{5} M / 32 \approx 9 \mathrm{MeV}$ & $V \tilde{V}$ & $28.4 \alpha_{2}^{6} \alpha_{\mathrm{em}} M \approx 3.6 \mathrm{keV}$ & ${ }^{1} s_{3} \gamma$ \\
\hline${ }_{J}^{2} p_{1}^{++}$ & 2 & 1 & 1 & 1 & $19.7 \mathrm{GeV}$ & $\mathcal{O}\left(\alpha_{2}^{7} M\right) \sim \mathrm{keV}$ & $V V$ & $20.4 \alpha_{2}^{4} \alpha_{\mathrm{em}} M \approx 2.5 \mathrm{MeV}$ & ${ }^{1} s_{3} \gamma$ \\
\hline${ }_{1}^{2} p_{3}^{+-}$ & 2 & 3 & 0 & 1 & $12 \mathrm{GeV}$ & $\mathcal{O}\left(\alpha_{2}^{8} M\right) \sim 10 \mathrm{eV}$ & $V V V$ & $\left(30.2 \alpha_{2}+0.3 \alpha_{\mathrm{em}}\right) \alpha_{2}^{4} M \approx 15.3 \mathrm{MeV}$ & ${ }^{1} s_{1+5} V$ \\
\hline${ }_{J}^{2} p_{5}^{++}$ & 2 & 5 & 1 & 1 & $2.2 \mathrm{GeV}$ & $\mathcal{O}\left(\alpha_{2}^{7} M\right) \sim \mathrm{keV}$ & $V V$ & $4.7 \alpha_{2}^{4} \alpha_{\mathrm{em}} M \approx 0.6 \mathrm{MeV}$ & ${ }^{1} s_{3} \gamma$ \\
\hline${ }_{1}^{3} s_{1}^{-+}$ & 3 & 1 & U & 0 & $3.8 \mathrm{GeV}$ & $120 \alpha_{2}^{5} M \approx 60 \mathrm{MeV}$ & $V \tilde{V}$ & $0.34 \alpha_{2}^{4} \alpha_{\mathrm{em}} M \approx 42 \mathrm{keV}$ & ${ }^{2} p_{3} \gamma$ \\
\hline${ }_{1}^{3} s_{3}^{--}$ & 3 & 3 & 1 & 0 & $1.7 \mathrm{GeV}$ & $15625 \alpha_{2}^{5} M / 1296 \approx 6.0 \mathrm{MeV}$ & $f_{L} \bar{f}_{L}+H H^{*}$ & $(0.003+0.005) \alpha_{2}^{4} \alpha_{\mathrm{em}} M \approx 1 \mathrm{keV}$ & ${ }^{2} p_{1+5} \gamma$ \\
\hline${ }_{1}^{3} s_{5}^{-+}$ & 3 & 5 & 0 & 0 & $1.7 \mathrm{MeV}$ & $21 \alpha_{2}^{5} M / 4 \approx 2.7 \mathrm{MeV}$ & $V \tilde{V}$ & $0.3 \alpha_{2}^{4} \alpha_{\mathrm{em}} M \approx 36 \mathrm{keV}$ & ${ }^{2} p_{3} \gamma$ \\
\hline${ }_{J}^{3} d_{3}^{--}$ & 3 & 3 & 1 & 2 & $0.9 \mathrm{GeV}$ & $\mathcal{O}\left(\alpha_{2}^{9} M\right) \sim \mathrm{eV}$ & $f_{L} \bar{f}_{L}$ & $0.4 \alpha_{2}^{4} \alpha_{\mathrm{em}} M \approx 52 \mathrm{keV}$ & ${ }^{2} p_{1+5} \gamma$ \\
\hline
\end{tabular}

Table 1. Main bound states of fermion weak 5 -plets with $M \approx 14 \mathrm{TeV}$. The parity $P=(-1)^{\ell+1}$ and charge conjugation $C=(-1)^{\ell+S}$ quantum numbers of bound states are broken by chiral weak gauge interactions to SM fermions. Hyper-fine components with different values of $J$ have the same decay rate. Decay rates are not $\mathrm{SU}(2)_{L}$-invariant because $W, Z$ emission is sometimes blocked by phase space; we report decay rates averaged over the weak components of bound states.

fermions with rate $\Gamma_{\text {ann }, f}=625 M / 2 n^{3}=0.96 \Gamma_{\text {ann }}$. The other vector triplet, the ${ }_{1}^{2} p_{3}^{+-}$ bound state with $S=0, \ell=1$, has opposite parity and annihilates in $V V V$ rather than in fermions. The bound state ${ }_{J}^{3} d_{3}^{--}$with $\ell=2$ has the right quantum numbers, but annihilation rates of bound states with $\ell>0$ are suppressed by extra powers of $\alpha_{2}$.

Table 1 also shows the decay widths among bound states: their computation will be discussed in section 3.3, where we discuss the associated collider signals.

\section{$3 \quad$ Bound-state production at colliders}

All bound states have narrow total width, $\Gamma=\Gamma_{\text {ann }}+\Gamma_{\text {dec }} \ll M$. Then, their collider phenomenology is well approximated $\grave{a}$ la Breit-Wigner such that their decay widths determine their production rates. The cross section for $s$-channel production is

$$
\sigma\left(i_{1} i_{2} \rightarrow B \rightarrow f\right) \approx \mathrm{BW}(s) \sigma_{\text {peak }}
$$

where

$$
\mathrm{BW}(s)=\frac{M_{B}^{2} \Gamma_{B}^{2}}{\left(s-M_{B}^{2}\right)^{2}+M_{B}^{2} \Gamma_{B}^{2}} \simeq \Gamma_{B} M_{B} \pi \delta\left(s-M_{B}^{2}\right), \quad \sigma_{\text {peak }}=\frac{16 \pi S_{B}}{M_{B}^{2} S_{i_{1}} S_{i_{2}}} \mathrm{BR}_{i_{1} i_{2}} \mathrm{BR}_{f}
$$

and $S_{i}$ is the spin times group multiplicity of the various particles (e.g. 2 for $\mu^{ \pm}, 3$ if $B$ is a vector singlet etc). 
The cross section needs to be convoluted with the energy distribution of a muon collider, described by some function $\wp(s)$ normalized as $\int \wp(s) d \sqrt{s}=1$. Assuming that each beam has a Gaussian energy distribution with standard deviation $\sigma_{E}$ one gets a Gaussian distribution

$$
\wp(s)=\frac{1}{\sqrt{2 \pi} \Delta_{E}} \exp \left[-\frac{\left(\sqrt{s}-M_{B}\right)^{2}}{2 \Delta_{E}^{2}}\right], \quad \Delta_{E}=\sqrt{2} \sigma_{E} .
$$

The energy resolution of a muon collider is expected to be $\sigma_{E} \approx 10^{-3} E \sim 14 \mathrm{GeV}$ [19], larger than the widths of bound states, $\Gamma_{B} \lesssim \mathrm{GeV}$. Thereby a muon collider cannot sit at the peak of the resonances, where the cross section is as large as allowed by unitarity. In the limit $\sigma_{E} \gg \Gamma_{B}$ the convoluted cross section is

$$
\sigma(i \rightarrow B \rightarrow f) \simeq \epsilon \sigma_{\text {peak }}, \quad \epsilon=\frac{\sqrt{\pi} \Gamma_{B}}{4 \sigma_{E}}
$$

Thanks to the $\sigma_{E}$ at the denominator, bound states that can be directly produced from $i_{1} i_{2}=\mu^{-} \mu^{+}$collisions can have cross sections comparable or bigger than tree-level SM cross sections, $\sigma \approx 4 \pi \alpha_{2}^{2} / s$.

\subsection{Production of ${ }^{n} s_{3}$ bound states from $\mu^{-} \mu^{+}$collisions}

We here study states that can be directly produced from $\mu^{-} \mu^{+}$collisions with a resonant $s-$ channel cross section. These are the states with the same quantum numbers as electroweak vectors: $I=3, S=1$ and $P C=--$, achieved in view of the constituent fermion 5-plets $\mathcal{X}$. The first such state is ${ }_{J=1}^{n=1} s_{I=3}^{--}\left({ }^{1} s_{3}\right.$ for short $)$, that exists for $M \gtrsim 4.4 \mathrm{TeV}$. Table 1 shows that, for $M=14 \mathrm{TeV},{ }^{n} s_{3}$ bound states exist for $n=\{1,2,3\}$. The leading-order cross section for $s$-channel production of their neutral component $B^{0}$ is given by eq. (3.4) with

$$
\epsilon \approx \frac{1}{192 n^{3}} \frac{10^{-3}}{\sigma_{E} / E}, \quad \sigma_{\text {peak }}\left(\mu^{+} \mu^{-} \rightarrow B_{1 s_{3}}^{0} \rightarrow f \bar{f}\right)=\frac{3 \pi}{M^{2}} \mathrm{BR}_{\mu} \mathrm{BR}_{f} \approx 30 \mathrm{fb} \frac{\mathrm{BR}_{f}}{\mathrm{BR}_{\ell}}
$$

where $\mathrm{BR}_{\ell}=1 / 25$ for any lepton flavour, and $\mathrm{BR}_{q}=3 / 25$ for any quark flavour. The denominator is 25 (rather than 24 ), taking into account the $1 / 25$ branching ratio into the Higgs multiplet. A more precise evaluation includes higher order effects. In particular, the signal cross section gets reduced by about a factor 2 taking into account initial state radiation (ISR) of $\gamma$ and $Z$. We perform MonteCarlo simulations by approximating such bound states as vectors $B_{n \mu}^{a}$ coupled as $g_{n} B_{n \mu}^{a}\left(\bar{f} \gamma_{\mu} T^{a} f\right)_{L}$ to left-handed SM fermions, and choosing couplings $g_{n}$ that reproduce the bound-state widths. Then, numerical results from MAdGraph [20] show that $\gamma$ radiation dominates. Such effect is analytically approximated by assigning a parton distribution function to each muon beam, such that the amount of muons with energy equal to the beam energy gets reduced by an order unity factor, analytically given by $\sim(\Gamma / M)^{4 \alpha_{\mathrm{em}} \ln \left(E / m_{\mu}\right) / \pi}$ [22]. Precise analytical results [21] agree with numerical results. 


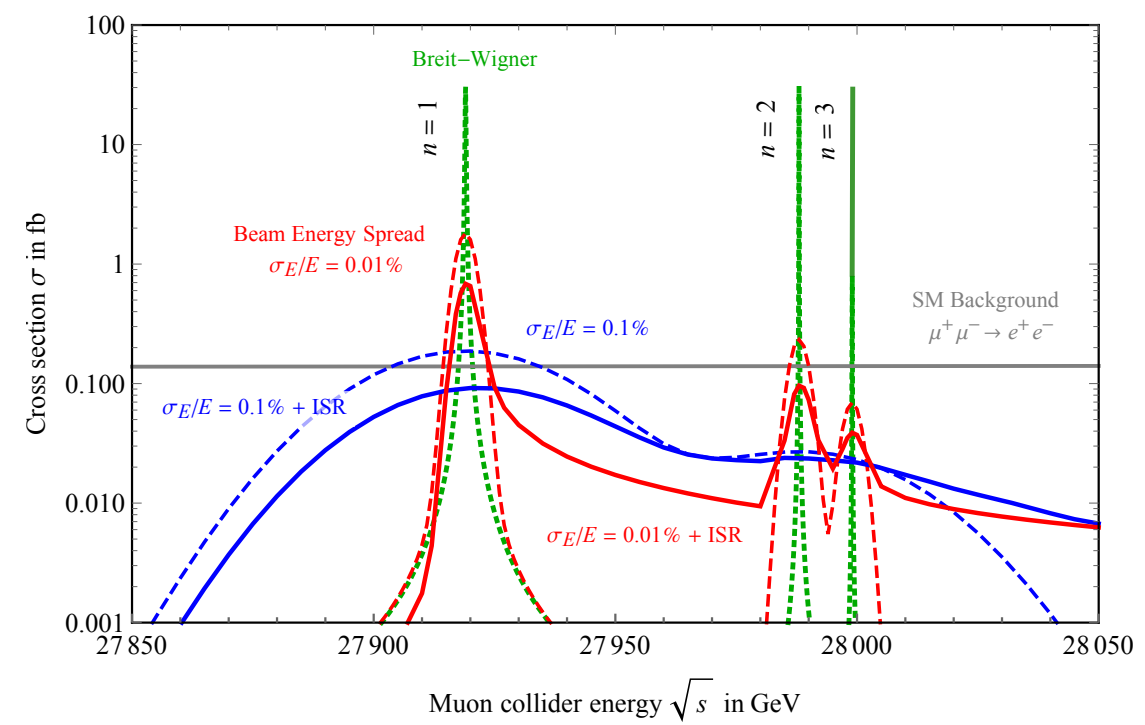

Figure 2. Bound-state signals of a Minimal Dark Matter 5-plet with constituent mass $M=14 \mathrm{TeV}$. The dotted green curves show the signal cross section for production of ${ }^{n} s_{1}$ DM bound states with $n=\{1,2,3\}$, ignoring the beam energy spread. The dashed curves show the signal cross section, for two different values of the beam energy spread, $\sigma_{E}=10^{-3} E$ (baseline value) and $\sigma_{E}=10^{-4} E$ (feasible value). The continuous curves show the signal cross section after also taking into account initial state emission. The gray horizontal curve is the $\mathrm{SM} \mu^{+} \mu^{-} \rightarrow e^{+} e^{-}$background.

Considering, for example, the $e^{-} e^{+}$final state (so that calorimeters can precisely measure their large energy), the SM background is

$\sigma_{\mathrm{SM}}\left(\mu^{+} \mu^{-} \rightarrow e^{+} e^{-}\right)=\frac{4 \pi \alpha_{\mathrm{em}}^{2}}{3 s}+\frac{2 \pi \alpha_{\mathrm{em}} \alpha_{2}}{3 c_{\mathrm{W}}^{2} s}\left(g_{L}+g_{R}\right)^{2}+\frac{\pi \alpha_{2}^{2}}{3 c_{\mathrm{W}}^{4} s}\left(g_{L}^{2}+g_{R}^{2}\right)^{2} \approx 140 \mathrm{ab} \frac{(28 \mathrm{TeV})^{2}}{s}$

where $s \gg M_{Z}^{2}, c_{\mathrm{W}}=M_{W} / M_{Z}, g_{L}=1 / 2-c_{\mathrm{W}}^{2}, g_{R}=1-c_{\mathrm{W}}^{2}$. We see that $\sigma_{\text {peak }}$ is 200 times larger than $\sigma_{\mathrm{SM}}$ (green dotted curve in figure 2 ) and that a design energy spread reduces it by $\epsilon \sim 1 / 200$ for $n=1$, providing a DM signal at the level of total SM backgrounds (dashed blue curve in figure 2 ). The $n=1$ state can be mildly separated from those with $n=\{2,3\}$, that have rates below the SM background and thereby need some dedicated search.

Figure 3a shows that the integrated luminosity needed to discover such state corresponds to about one day of running, taking into account its annihilation channels into $e^{+} e^{-}$and jets and without performing selection cuts (for simplicity, we do not include annihilations into $\mu^{+} \mu^{-}$, which have a larger background due to $t$-channel vector exchange that can be efficiently reduced by cuts on $p_{T}$ and other variables). We assumed a $70 \%$ efficiency for detecting each electron or jet in the final state. Reducing the beam energy spread reduces the needed integrated luminosity, but by an amount similar to the expected loss in collider luminosity.

With a feasible reduction of $\sigma_{E}$ by one order of magnitude, the cross section for producing the ${ }^{1} s_{3}$ bound state becomes one order of magnitude larger than SM backgrounds, and the excited bound states with $n=2,3$ can be separated and acquire total cross sections 


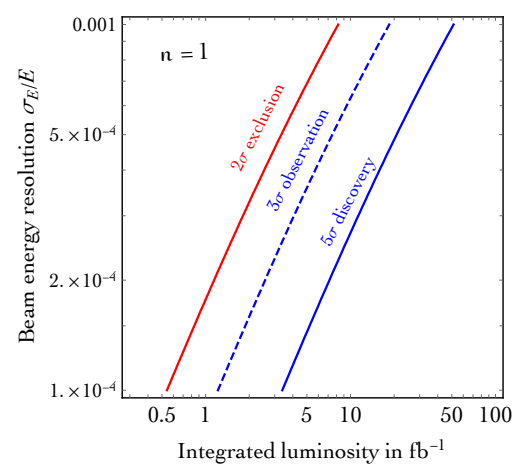

(a)

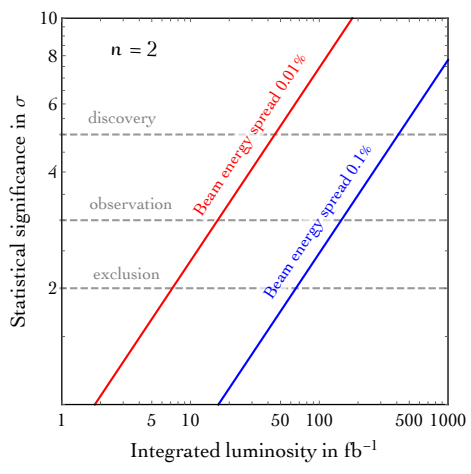

(b)

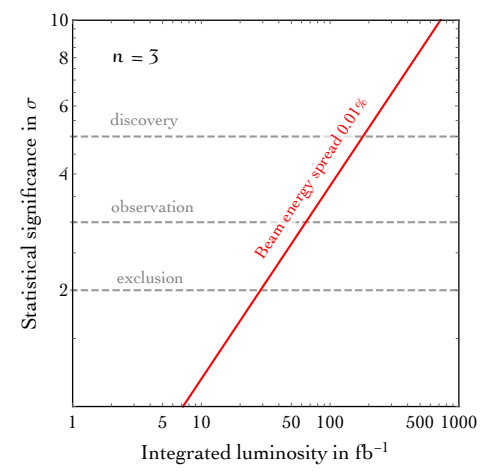

(c)

Figure 3. The integrated luminosity needed to see the ${ }^{n} s_{3}$ bound states with mass $2 M=28 \mathrm{TeV}$ of a Minimal DM 5-plet at a muon collider with $s \approx 4 M^{2}$ is much smaller than the possible value, $90 / \mathrm{ab}$ at $\sigma_{E} / E=10^{-3}$.

at the level of the SM backgrounds (dashed red curve in figure 2). After taking initial state radiation into account, one obtains the continuous curves in figure 2 , where peaks become asymmetric and larger above the threshold due to the 'radiative return' phenomenon. Figure 3b,c show the integrated luminosity needed to discover such states. The non-resonant loop corrections considered by [11] at $\sqrt{s}$ slightly above the $2 M$ threshold interfere destructively with the SM background leading to a decrease of the SM cross section by up to $8 \%$.

The charged components $B_{\mu}^{ \pm}$of the isospin triplet of bound states are produced with a relatively large cross section, given that the partonic neutrino component of a $\mu^{ \pm}$beam is peaked at energy fraction $x=1$ [23], in view of soft $W^{ \pm}$emission. By running a bit above the peak, the state with $n=1$ is produced as $\mu^{+} \mu^{-} \rightarrow B_{\mu}^{ \pm} W_{\mu}^{\mp}$ with fb-scale cross section, as shown by the blue curve in figure 4 .

Finally, we mention that the state ${ }^{3} d_{3}$ too has the same quantum numbers as electroweak vectors and can thereby be produced directly from $\mu^{+} \mu^{-}$collision; however its annihilation rate (see bottom row of table 1 ) is highly suppressed by $\alpha_{2}^{5+2 \ell}$ in view of $\ell=2$ and we neglect it.

\subsection{Production of other bound states from $V V$ collisions}

The other bound states annihilate to weak vectors and can thereby be produced through associated production via vectors. Then, the energy spread in the effective collision energy becomes large, $\sigma_{E} \sim \sqrt{s}$, and the cross sections small. These more general processes can be computed using automated codes [20], approximating bound states as particles with effective couplings to their decay products that reproduce the widths [24] computed in table 1. For example, the ground pseudo-scalar bound state ${ }^{1} s_{1}$ with $I=1$ can be written as a scalar singlet $B$ coupled as $B \epsilon_{\mu \nu \mu^{\prime} \nu^{\prime}} V_{\mu \nu}^{a} V_{\mu^{\prime} \nu^{\prime}}^{a}$. The pseudo-scalar bound state ${ }^{1} s_{5}$ with $I=5$ can be written as a scalar $B_{a a^{\prime}}$ in the symmetric trace-less representation of $\mathrm{SU}(2)_{L}$ coupled as $B_{a a^{\prime}} \epsilon_{\mu \nu \mu^{\prime} \nu^{\prime}} V_{\mu \nu}^{a} V_{\mu^{\prime} \nu^{\prime}}^{a^{\prime}}$. 

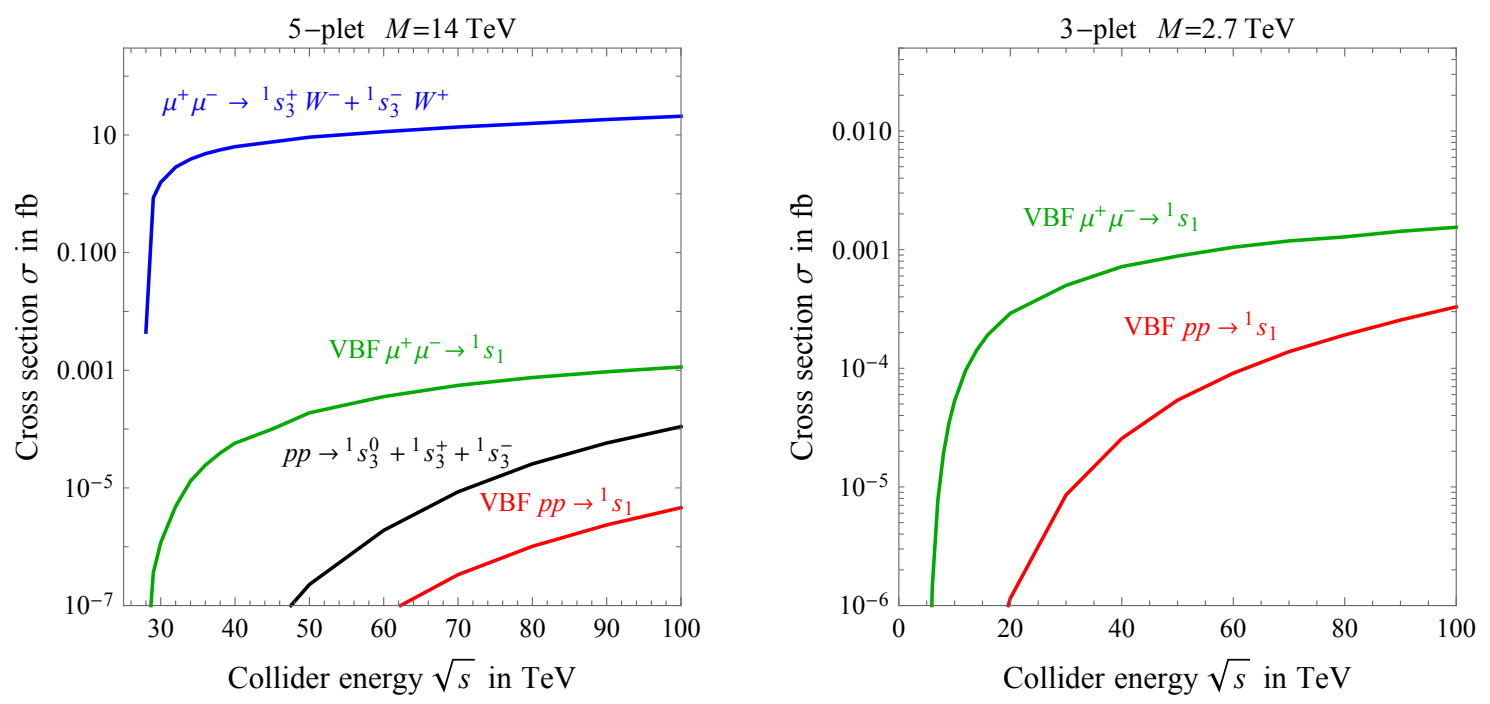

Figure 4. Cross sections for the production of some bound states of the Minimal DM fermionic 5-plet with constituent mass $M=14 \mathrm{TeV}$ (left) and of the fermionic 3-plet with $M=2.7 \mathrm{TeV}$ (right) at a $\mu^{+} \mu^{-}$collider and at a $p p$ collider.

We focus on the ground state ${ }^{1} s_{1}$, as it has the largest annihilation rate. It can be produced via scatterings of SM electroweak vectors, $\gamma \gamma \rightarrow B_{1 s_{1}}, \gamma \mu^{ \pm} \rightarrow B_{1 s_{1}} \mu^{ \pm}, \mu^{+} \mu^{-} \rightarrow$ $B_{1 s_{1}} \mu^{+} \mu^{-}, \mu^{+} \mu^{-} \rightarrow B_{1 s_{1}} \nu_{\mu} \bar{\nu}_{\mu}$. Resonant production is not possible and one thereby must run at higher $\sqrt{s}>2 M$ : the green curve in figure 4 shows that, as expected, the production cross section is much smaller.

The red curve in figure 4 shows its analogous production cross sections at a $p p$ collider, which is even smaller given that vector partons in a $p$ beam have lower energy than in a $\mu$ beam. For completeness, the black curve in figure 4 shows the cross section for production at a $p p$ collider of the ${ }^{1} s_{3}$ bound state discussed in the previous section. We do not discuss the backgrounds.

Furthermore, we consider a Wino-like Minimal DM fermionic triplet. The DM abundance is reproduced thermally for $M=2.7 \mathrm{TeV}$. At this mass only one ${ }^{1} s_{1}$ bound state exists with $E_{B} \approx 68 \mathrm{MeV}$ and $\Gamma_{B}=8 \alpha_{2}^{5} M \approx 4 \mathrm{MeV}$ [2]. This bound state cannot be produced with a resonantly-enhanced cross section. Figure $4 \mathrm{~b}$ shows its production cross section at a muon or $p p$ collider.

\subsection{Decays of bound states and their collider signals}

In this section we describe the computation of the bound state decays listed in table 1 , having in mind that we seek characteristic collider signals produced by decays among bound states. The leading-order decays $B \rightarrow B^{\prime} V$ proceed through the emission of a weak vector boson $V$, which is often a photon as the phase space for $W, Z$ emission is often closed. Such process dominantly occurs via electric dipole transitions, although magnetic dipole transitions happen to be important in cases where selection rules forbid electric dipole transitions. We compute bound states in the $\mathrm{SU}(2)_{L}$-symmetric approximation, so that 
bound states of two 5 -plets have two indices $i j$ in the 5 representation, that can be converted into isospin eigenstates $B_{\tilde{I} \tilde{I}_{3}}$ through Clebsch-Gordan coefficients: $B_{i j}=C_{i j}^{\tilde{I} \tilde{I}_{3}} B_{\tilde{I} \tilde{I}_{3}}$.

- The effective interaction hamiltonian for the electric dipole at leading order is

$$
H_{\mathrm{el}}=-\frac{g_{2}}{M}\left[\vec{A}^{a}\left(x_{1}\right) \cdot \vec{p}_{1} T_{i^{\prime} i}^{a} \delta_{j j^{\prime}}+\vec{A}^{a}\left(x_{2}\right) \cdot \vec{p}_{2} \bar{T}_{j^{\prime} j}^{a} \delta_{i i^{\prime}}\right]+g_{2} \alpha_{2}\left[\vec{A}^{a}(0) \cdot \hat{r}\right] T_{i^{\prime} i}^{b} \bar{T}_{j^{\prime} j}^{c} f^{a b c}
$$

leading to the following selection rules: $|\Delta \tilde{I}|=1,|\Delta \ell|=1, \Delta S=0$. Decay rates are obtained as

$$
\begin{aligned}
& \Gamma\left({ }^{2} p_{\tilde{I}} \rightarrow{ }^{1} s_{\tilde{I}^{\prime}}+V^{a}\right)=\frac{16}{9 I_{2 p}} \frac{\alpha_{2} k}{M^{2}} \sum_{\tilde{I}_{3} \tilde{I}_{3}^{\prime}}\left|\int r^{2} \mathrm{~d} r R_{\tilde{I}_{, 2 p}}\left(C_{\mathcal{J}}^{a \tilde{I}_{3} \tilde{I}_{3}^{\prime}} \partial_{r}-C_{\mathcal{T}}^{a \tilde{I}_{3} \tilde{I}_{3}^{\prime}} \frac{\alpha_{2} M}{2}\right) R_{\tilde{I}^{\prime}, 1 s}\right|^{2} \\
& \Gamma\left({ }^{3} s_{\tilde{I}} \rightarrow{ }^{2} p_{\tilde{I}^{\prime}}+V^{a}\right)=\frac{16}{3 I_{3 s}} \frac{\alpha_{2} k}{M^{2}} \sum_{\tilde{I}_{3} \tilde{I}_{3}^{\prime}}\left|\int r^{2} \mathrm{~d} r R_{\tilde{I}^{\prime}, 2 p}\left(C_{\mathcal{J}}^{a \tilde{I}_{3} \tilde{I}_{3}^{\prime}} \partial_{r}+C_{\mathcal{T}}^{a \tilde{I}_{3} \tilde{I}_{3}^{\prime}} \frac{\alpha_{2} M}{2}\right) R_{\tilde{I}, 3 s}\right|^{2}
\end{aligned}
$$

where $r$ is the radius, $R(r)$ are normalized radial wave-functions, $k$ is the spatial momentum of $V, I$ is the isospin of the initial bound state, and

$$
C_{\mathcal{J}}^{a \tilde{I}_{3} \tilde{I}_{3}^{\prime}}=\frac{1}{2} \operatorname{Tr}\left[C^{\tilde{I}^{\prime} \tilde{I}_{3}^{\prime}}\left\{C^{\tilde{I} \tilde{I}_{3}}, T^{a}\right\}\right], \quad C_{\mathcal{T}}^{a \tilde{I}_{3} \tilde{I}_{3}^{\prime}}=i \operatorname{Tr}\left[C^{\tilde{I}^{\prime} \tilde{I}_{3}^{\prime}} T^{b} C^{\tilde{I} \tilde{I}_{3}} T^{c}\right] f^{a b c} .
$$

- The effective interaction hamiltonian for the magnetic dipole at leading order is (see e.g. [25])

$$
H_{\text {mag }}=-\frac{g_{2}}{2 M}\left[T_{i^{\prime} i}^{a} \delta_{j j^{\prime}} \vec{\sigma} \cdot \vec{B}^{a}\left(x_{1}\right)+\bar{T}_{j^{\prime} j}^{a} \delta_{i i^{\prime}} \vec{\sigma} \cdot \vec{B}^{a}\left(x_{2}\right)\right]+\cdots
$$

leading to the following selection rules: $|\Delta \tilde{I}|=1, \Delta \ell=0,|\Delta S|=1$. Decay rates are obtained as [25]

$$
\Gamma\left({ }^{n_{i}} s_{\tilde{I}_{i}} \rightarrow{ }^{n_{f}} s_{\tilde{I}_{f}}+V^{a}\right)=\frac{2^{3}}{I_{i}} \frac{\alpha_{2} k^{3}}{M^{2}} \sum_{\tilde{I}_{3, i} \tilde{I}_{3, f}}\left|C_{\mathcal{J}}^{a \tilde{I}_{3, i} \tilde{I}_{3, f}} \int r^{2} \mathrm{~d} r R_{n_{i} s_{\tilde{I}_{i}}} R_{n_{f} s_{\tilde{I}_{f}}}\right|^{2}
$$

with no contribution from the omitted non-abelian term in eq. (3.11).

- Higher-order interactions lead to multiple-vector emission, with suppressed rates that turn out to be negligible.

As discussed in section 3.1, the lightest bound state that can be produced resonantly is the neutral component of ${ }^{1} s_{3}$. This is the only component of ${ }^{1} s_{3}$ that can decay $\left(W^{ \pm}\right.$ emission from charged components of ${ }^{1} s_{3}$ is kinematically blocked) to ${ }^{1} s_{1} \gamma$ via a magnetic transition with a rate $\Gamma_{\mathrm{dec}}=3 \times 4.6 \mathrm{keV}$. Such rate is of order $\alpha_{2}^{6} \alpha_{\mathrm{em}} M$, where an $\alpha_{2}^{2}$ factor arises from the $\gamma$ phase space; another $\alpha_{2}^{4}$ from the magnetic field $\vec{B}$; the $\alpha_{\mathrm{em}}$ from photon emission. Taking into account its annihilation rate, the neutral component of the ${ }^{1} s_{3}$ bound state decays into a monochromatic $\gamma$ with energy $E_{\gamma} \approx 38 \mathrm{GeV}$ with branching ratio $\mathrm{BR}_{\mathrm{dec}} \approx 910^{-5}$. This corresponds to 19 events in a run with baseline $\sigma_{E}=10^{-3}$ and luminosity $\mathcal{L}=90 /$ ab.

Higher order states are produced with a lower cross section, that scales as $\Gamma_{\text {ann }} \propto$ $1 / n^{3}$. Nevertheless, such states could give a higher rate of decay events, proportional to $\sigma \mathrm{BR}_{\mathrm{dec}} \propto \Gamma_{\mathrm{ann}} \times \Gamma_{\mathrm{dec}} / \Gamma_{\mathrm{ann}} \propto \Gamma_{\mathrm{dec}}$. 
- At $n=2$, the ${ }^{2} s_{3}$ bound state similarly decays magnetically, with the difference that it can now also emit massive weak bosons, and decay into multiple states ${ }^{1} s_{1},{ }^{1} s_{5}$ (we neglect decays in ${ }^{2} s_{1}$ because their rate is negligibly small, at eV level). The neutral component of ${ }^{2} s_{3}$ decays emitting a $\gamma$ with rate $\Gamma_{\text {dec }} \approx 2.0 \mathrm{keV}$ and emitting a $Z$ with rate $\Gamma_{\mathrm{dec}} \approx 1.7 \mathrm{keV}$; charged components have similar decay rates. In view of the lower binding energy and wave-function overlap, ${ }^{2} s_{3}$ thereby gives a similar number of decay events as ${ }^{1} s_{3}$. As a result, the $\gamma$ decays of the ${ }^{2} s_{3}$ neutral component produces two distinctive single-photon lines, at $E_{\gamma} \approx 105 \mathrm{GeV}$ and $13 \mathrm{GeV}$, as well as $Z$ bosons.

- At $n=3$, the ${ }^{3} s_{3}$ bound state can decay electrically into ${ }^{2} p_{1+5} \gamma$, with a rate of order $\alpha_{2}^{4} \alpha_{\mathrm{em}} M$ (where an $\alpha_{2}^{2}$ factors arises from the $\gamma$ phase space; another $\alpha_{2}^{2}$ from the dipole matrix element; the $\alpha_{\mathrm{em}}$ from photon emission). The numerical coefficient turns however to be small, and the decay rate is again around a keV. More precisely, only the neutral component can decay into ${ }^{2} p_{1}$, and all components decay equally into ${ }^{2} p_{5}$. Thereby, table 1 implies that the decay rate of the neutral component is $\Gamma_{\text {dec }}=(3 \times 0.003+$ $0.005) \alpha_{2}^{4} \alpha_{\mathrm{em}} \approx 1.7 \mathrm{keV}$. The ${ }^{2} p_{1+5}$ bound states next dominantly decay via a large electric dipole into ${ }^{1} s_{3}$, that annihilates. This process thereby gives a set of multiplephoton lines, with $E_{\gamma} \approx\{18 \mathrm{GeV}, 60 \mathrm{GeV}\}$, and with $E_{\gamma} \approx\{0.5 \mathrm{GeV}, 79 \mathrm{GeV}\}$. As signal events have very distinctive $\gamma \gamma$ signatures, backgrounds can be strongly reduced.

For completeness, in table 1 we also computed decay rates of other states that cannot be produced resonantly with large rates. Thereby we do not discuss them. All above numbers assume $M=14 \mathrm{TeV}$ and need to be recomputed otherwise.

\section{Conclusions}

We considered DM as electroweak multiplets, and studied the effects of their electroweak bound states at future colliders. We found that bound states with the same quantum numbers as electroweak vectors can be produced resonantly with large cross sections by running lepton colliders at the appropriate $\sqrt{s}$. Such bound states exist if DM is a heavy enough fermionic multiplet. ${ }^{3}$ A wino-like weak fermionic triplet with 'thermal' mass that reproduces the cosmological DM density, $M=2.7 \mathrm{TeV}$, does not form such bound states. Three of such bound states arise if DM is an automatically-stable fermionic weak 5-plet with 'thermal' mass $M \approx 14 \mathrm{TeV}$. The level structure is plotted in figure 1 , the main properties of the bound states are computed in table 1 , and figure 2 shows that the three predicted peaks would be easily observable at a muon collider, running at $\sqrt{s} \approx 2 M$ and with a beam energy spread $\sigma_{E} / E=10^{-3}$ or better. The production rates of the neutral components $B_{n}^{0}$ of the bound-state triplets are so large that one day of running may be enough for discovery, see figure 3. One could next search for rarer but more characteristic sets of single and multiple lines $\gamma$ produced by decays among bound states, as discussed in section 3.3. The charged components $B_{n}^{ \pm}$of the bound state triplets can also be produced

\footnotetext{
${ }^{3}$ A similar resonant enhancement arises from bound states with the same quantum numbers as the Higgs boson, possibly present in speculative DM models where the Higgs mediates attractive forces between DM constituents, fermionic or bosonic.
} 
with partially enhanced cross section at a broader $\sqrt{s} \gtrsim 2 M$, as neutrinos have a peaked partonic distribution in muons.

We also studied the other bound states that cannot be produced resonantly. The extra 5-plet bound states in table 1, as well as the only bound state formed by a 3-plet, are produced at $\mu^{+} \mu^{-}$or $p p$ colliders with small non-resonant cross sections as shown in figure 4 .

As an aside final comment, we mention a new Minimal DM signal even more futuristic than a muon collider. ${ }^{4} \mathrm{DM} \mathcal{X}^{0}$ gravitationally attracted by a neutron star reaches relativistic velocity before hitting its surface, so that charged current scatterings such as $\mathcal{X}^{0} n \rightarrow$ $\mathcal{X}^{-} p$ and $\mathcal{X}^{0} p \rightarrow \mathcal{X}^{+} n$ become kinematically allowed despite the $\Delta M \approx 166 \mathrm{MeV}$ gap, and have a large tree-level cross section $\sigma \sim m_{n}^{2} / v^{4} \sim 10^{-38} \mathrm{~cm}^{2}$. Decays of charged components can then produce neutrinos with energy around $10 \mathrm{MeV}$ at rate $\dot{N}_{\nu} \sim \dot{M} / \Delta M \sim 10^{25} / \mathrm{sec}$. Here $\dot{M}_{\mathrm{DM}}=\rho_{\mathrm{DM}} v_{\mathrm{DM}} \pi b^{2}$ is the DM mass that falls in the neutron star. The impact parameter is $b / R_{\mathrm{ns}}=v_{\mathrm{esc}} / v_{\mathrm{DM}} / \sqrt{1-v_{\mathrm{esc}}^{2}}$ where $R_{\mathrm{ns}} \sim\left(M_{\mathrm{Pl}} / m_{n}\right)^{3} \sim$ few $\mathrm{km}$ is the radius of the neutron star, and $v_{\text {esc }} \sim 1$ is the escape velocity. Given that the nearest neutron stars are expected to be at distance $d \sim$ pc from the Earth, the resulting neutrino flux $\Phi_{\nu} \sim \dot{N}_{\nu} / 4 \pi d^{2}$ is about 10 orders of magnitude below the expected background of supernova neutrinos. A very futuristic detector close to a neutron star is needed to reveal the signal.

\section{Acknowledgments}

We thank Michele Redi, Andrea Wulzer and Ivano Basile. This work was supported by the ERC grant 669668 NEO-NAT and by PRIN 2017FMJFMW.

Open Access. This article is distributed under the terms of the Creative Commons Attribution License (CC-BY 4.0), which permits any use, distribution and reproduction in any medium, provided the original author(s) and source are credited.

\section{References}

[1] M. Cirelli, N. Fornengo and A. Strumia, Minimal dark matter, Nucl. Phys. B 753 (2006) 178 [hep-ph/0512090] [INSPIRE].

[2] A. Mitridate, M. Redi, J. Smirnov and A. Strumia, Cosmological Implications of Dark Matter Bound States, JCAP 05 (2017) 006 [arXiv: 1702.01141] [INSPIRE].

[3] J. Hisano, K. Ishiwata and N. Nagata, QCD Effects on Direct Detection of Wino Dark Matter, JHEP 06 (2015) 097 [arXiv: 1504.00915] [INSPIRE].

[4] T. Golling et al., Physics at a $100 \mathrm{TeV}$ pp collider: beyond the Standard Model phenomena, arXiv: 1606.00947 [INSPIRE].

[5] M. Cirelli, F. Sala and M. Taoso, Wino-like Minimal Dark Matter and future colliders, JHEP 10 (2014) 033 [Erratum ibid. 01 (2015) 041] [arXiv: 1407.7058] [INSPIRE].

[6] B. Ostdiek, Constraining the minimal dark matter fiveplet with LHC searches, Phys. Rev. D 92 (2015) 055008 [arXiv: 1506.03445] [INSPIRE].

\footnotetext{
${ }^{4}$ A.S. thanks Paolo Panci and Raghuveer Garani for pointing it out.
} 
[7] T. Han, S. Mukhopadhyay and X. Wang, Electroweak Dark Matter at Future Hadron Colliders, Phys. Rev. D 98 (2018) 035026 [arXiv: 1805.00015] [inSPIRE].

[8] M. Saito, R. Sawada, K. Terashi and S. Asai, Discovery reach for wino and higgsino dark matter with a disappearing track signature at a $100 \mathrm{TeV}$ pp collider, Eur. Phys. J. C 79 (2019) 469 [arXiv: 1901.02987] [InSPIRE].

[9] T. Han, Z. Liu, L.-T. Wang and X. Wang, WIMPs at High Energy Muon Colliders, Phys. Rev. D 103 (2021) 075004 [arXiv: 2009.11287] [InSPIRE].

[10] R. Capdevilla, F. Meloni, R. Simoniello and J. Zurita, Hunting wino and higgsino dark matter at the muon collider with disappearing tracks, arXiv:2102.11292 [INSPIRE].

[11] T. Katayose, S. Matsumoto and S. Shirai, Non-perturbative Effects on Electroweakly Interacting Massive Particles at Hadron Collider, Phys. Rev. D 103 (2021) 095017 [arXiv: 2011.14784] [INSPIRE].

[12] W. Shepherd, T.M.P. Tait and G. Zaharijas, Bound states of weakly interacting dark matter, Phys. Rev. D 79 (2009) 055022 [arXiv:0901.2125] [InSPIRE].

[13] H. An, B. Echenard, M. Pospelov and Y. Zhang, Probing the Dark Sector with Dark Matter Bound States, Phys. Rev. Lett. 116 (2016) 151801 [arXiv:1510.05020] [INSPIRE].

[14] Y. Tsai, L.-T. Wang and Y. Zhao, Dark Matter Annihilation Decay at The LHC, Phys. Rev. D 93 (2016) 035024 [arXiv: 1511.07433] [inSPIRE].

[15] A. Krovi, I. Low and Y. Zhang, Broadening Dark Matter Searches at the LHC: Mono-X versus Darkonium Channels, JHEP 10 (2018) 026 [arXiv:1807.07972] [INSPIRE].

[16] L. Di Luzio, R. Gröber and G. Panico, Probing new electroweak states via precision measurements at the LHC and future colliders, JHEP 01 (2019) 011 [arXiv:1810.10993] [INSPIRE].

[17] Y. Tsai, T. Xu and H.-B. Yu, Displaced Lepton Jet Signatures from Self-Interacting Dark Matter Bound States, JHEP 08 (2019) 131 [arXiv:1811.05999] [INSPIRE].

[18] M. Cirelli, A. Strumia and M. Tamburini, Cosmology and Astrophysics of Minimal Dark Matter, Nucl. Phys. B 787 (2007) 152 [arXiv:0706.4071] [InSPIRE].

[19] J.P. Delahaye et al., Muon Colliders, arXiv:1901.06150 [INSPIRE].

[20] J. Alwall et al., The automated computation of tree-level and next-to-leading order differential cross sections, and their matching to parton shower simulations, JHEP 07 (2014) 079 [arXiv: 1405.0301] [INSPIRE].

[21] S. Jadach, B.F.L. Ward and Z. Was, Coherent exclusive exponentiation for precision Monte Carlo calculations, Phys. Rev. D 63 (2001) 113009 [hep-ph/0006359] [INSPIRE].

[22] M. Greco, T. Han and Z. Liu, ISR effects for resonant Higgs production at future lepton colliders, Phys. Lett. B 763 (2016) 409 [arXiv:1607.03210] [INSPIRE].

[23] T. Han, Y. Ma and K. Xie, High energy leptonic collisions and electroweak parton distribution functions, Phys. Rev. D 103 (2021) L031301 [arXiv:2007.14300] [INSPIRE].

[24] Y. Kats and M.D. Schwartz, Annihilation decays of bound states at the LHC, JHEP 04 (2010) 016 [arXiv: 0912.0526] [INSPIRE].

[25] R. Mahbubani, M. Redi and A. Tesi, Dark Nucleosynthesis: Cross-sections and Astrophysical Signals, JCAP 02 (2021) 039 [arXiv:2007.07231] [INSPIRE]. 